\title{
Repositories and Interoperability Standards
}

\author{
Hondjack Dehainsala $^{1}$ and Philippe Perdriau ${ }^{2}$ \\ ${ }^{1}$ AREVA, France as Interoperability Expert \\ ${ }^{2}$ EURIWARE, France as PLM project manager \\ hondjack. dehainsala@areava.com, \\ philippe.perdriau@euriware.fr
}

\begin{abstract}
Enterprise have needs for interoperability and exchange data interact with repositories; some standards exists and news are come in, particularly those related to the life cycle of facilities and plants. Technical and functional principles have been implemented in an interoperability platform built to meet these needs and to be open to the different standards (existing and news).

PLM tools for repository management are inadequate for handling exchanges in that they consider only the development of point-to-point tools or data copying, and that current exchange standards are also about point-to-point tools and do not really meet the needs of engineering companies and facility operators. This deficiency has led the community to focus on new standards based on Semantic Web technologies (in particular ISO15926) [8] which make these repositories and other authoring tools interoperable with one another.

Feedback is given on the inclusion of this standard, its implementation and deployment.
\end{abstract}

Keywords: PLM, Plants, ISO15926, Iring, Exchange Process, Interoperability, Semantic-Web, AIRE.

\section{The Need to Interoperate and the Expected Functions}

\subsection{Functions Integrated into the Repositories}

The term PLM is taken to mean all processes, activities and functions used to manage and configure data, documents, files and information during the cycle [7] of a product or facility. Presently only functions and data are considered.

PLMs are widely deployed in manufacturing industries [7] and are starting to be in companies involved in the design, construction, operation, maintenance and decommissioning of facilities. However, in this latter context special features appear that are particularly linked to the eco-system engaged on projects and to the nature of the objects and design processes of continuous-process industries.

\subsection{Purpose of This Document}

The purpose of this paper is to analyze the features and tools for data exchange and between systems to make them interoperable, given, to use the definition outlined by Wikipedia, that interoperability is the ability of a product or system, whose interfaces 
are fully known, to work with other existing or future products or systems with unrestricted access or implementation; the creation of a specific tool to make A and B communicate does not make A and B interoperable.

The main question is: do we need interoperability with PLM tools and why?

After that, we consider the characteristic of exchange standards and why the news standards like ISO15926 are based on new technology as the semantic Web.

We conclude with our feedback from a platform development and from first tests in interoperability.

\subsection{Special Cases in the Design, Construction and Maintenance of Petrochemical, Chemical and Nuclear Power Process Facilities}

In these situations, the eco-system of the stakeholders involved is not known in advance from one project to another, which distinguishes this context from those commonly encountered in other sectors. It is assembled and dismantled for each operation: partners one day are competitors the next and suppliers' cultural differences are important, as the projects are mainly international with the contractual obligation to employ local businesses.

Operators ultimately recover the documentation (data, study, construction, inspection and commissioning documents) models, files and requirements, and classify and integrate them into their own operational systems or archiving.

Facilities comprise a large number of objects (of the order of one million compared, for example, with a car which has only a few thousand).

At this stage, the following observation can be made:

- The exchanges between systems are numerous, very diverse and need to be controlled and secured [2]

- If we consider that out of the million objects referenced in a facility, $10 \%$ are subject to a collaborative process with the outside world and that this process requires 3-5 exchanges of partial or complete information, this makes about 1000 exchanges per day at the peak, with about a hundred stakeholders.

- A single repository shared with several stakeholders is not a good idea, in addition to the fact that it is very complex to set up (especially if it is needed throughout the duration of a project, because it may be completed only after the end of the project).

A functional architecture should therefore be chosen, suited to the needs of each entity and to its responsibilities, but open and interoperable with other systems.

Example: interoperability need for an operator

An operator uses different business views of the various systems and equipment items

- For example, a technological view per equipment family

- A maintenance view for equipment maintenance and monitoring activities

- An operational view for control and supervision systems

- A project view related to carrying out technical modifications

The PLM can produce different business views (trees and data), and present structures identical to the internal breakdown of the various tools in the information system. The same object can then be seen by the different tools while retaining the overall consistency, as shown in the diagram below. 


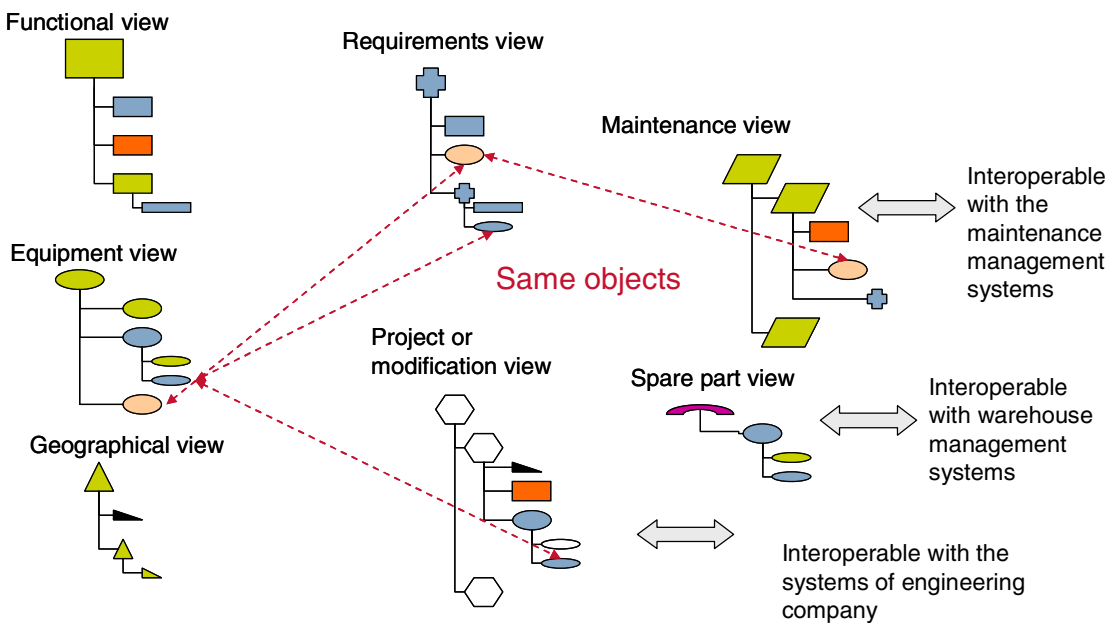

Fig. 1. Interoperability needs for an operator

The interoperability functions are then used to synchronize the systems, but it is also necessary to control the process of dissemination and integration between the source and the various targets.

\subsection{Exchange Needs with Others: How to Achieve This}

The previous sections show the diversity of exchange needs in both engineering projects and facility modification activities. There are structured into 4 categories:

\section{1) Exchanges with suppliers}

- Supplies input data to subcontractors

- Information back from supplier to populate the engineering databases

- Creation of collaborative catalogues

2) Coordination between non-integrated specialties

- Synchronizing data (periodic or with milestones) between unconnected systems

\section{3) Exchanges within a consortium}

- Controlling and synchronizing data (periodic or with milestones) between unconnected systems

\section{4) Providing data to a third-party}

- Periodic or with milestones = handover

- Populating operating and maintenance

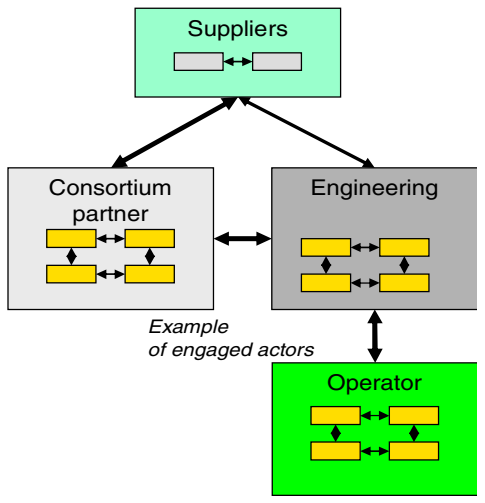

Fig. 2. Exchanges between Plant actors systems 
While some exchanges consist of synchronizing information, it is standard practice they are supported by the publishing processes on the one hand and integration processes on the other. It is recommended to filter and check the data to be made accessible, control the quality of the data received and see the changes; the tools must be able to deal with the exchange safely, both for the value of data and for a data set or the whole of a facility, either in sync mode or via formalized transmissions.

The lack of interoperability between these stakeholders is a source of costs $(4 \%$ according to [1]) which may be important in absolute terms in major contracts and may be even more so in relative terms for modifications to facilities, even if they are less costly.

In conclusion, interoperability functions are complementary functions that are found in PLM tools. The development of tools, based on exchange standards, connected to PLMs, but also to other sources of information, helps to streamline information systems and optimizes project processes. Only adopting a standard shared by the eco-system is able to solve the problem.

\section{Exchange or Interoperability Standards and New Technologies}

Exchange standards (ISO10303, ISO15926, for example) have similar characteristics:

1. An expression language of a data model

2. A data model representing a concept,

3. A dictionary describing types of reference objects and their characteristics,

4. Means to exchange and access information.

The choice of the exchange standard is based on the characteristics of the data model and the contents of the dictionary.

The usage of a standard by a company differs according to the disciplines (civil engineering, equipment, I \& $\mathrm{C}$, requirements), that these often refer the same information and that the consistency of information published or integrated becomes very difficult to maintain.

The ISO15926 standard (also called iRing) developed and maintained by the association PCA (POASC CAESAR ASSOCIATION) under the authority of the FIATECH (in relation with standardization bodies, in particular TC184/SC4) proposes a significant change in the approach in terms of both modelling and technology.

In terms of modelling, the following notable points can be distinguished:

1. The model is based on the overall classification if each element satisfies the inclusion criteria in its category and on class specialization for a subclass to inherit the properties of the parent class; relationships between classes can be also categorized.

2. This fairly complex modelling is intended to be described in the language of the Semantic Web, i.e. OWL (Ontology Web Language).

3. Objects and object attributes are identified by URIs (Uniform Resource Identifier) and allow sharing by means of web navigation. 
4. The ISO15926 standard, based on the notion of roles (defining attributes of a class) that can be instantiated on an object, enables representation to be simplified while remaining under the control of the principles set out in the general model.

5. Each company makes a mapping between the data model of its business application with the data model of ISO15926, its dictionary (private or public) and its roles (templates).

In terms of technology, using the Semantic Web [3] provides the following benefits:

1. Based on a general purpose, non-specific communication standard for technical data: this makes it possible to use distributed technological layers.

- So OWL modelling can be handled by existing tools inherent in this technology.

2. Using rules, this handling makes it possible to combine different concepts (domains) insofar as a relationship can be expressed to relate these concepts.

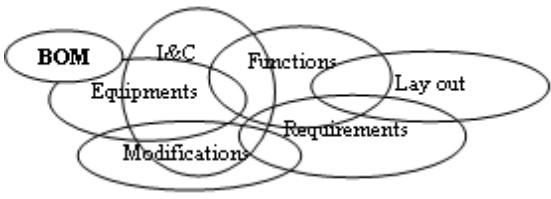

Fig. 3. Example of adding different concepts

The dictionary of standard ISO15926 (RDL) today contains the characteristics of objects contained in the facility: equipment (heat exchangers, columns, etc.), components (pumps, valves, motors, etc.) which make it possible to describe a technical tree of physical objects. It is also possible to describe an abstract object tree as a functional tree.

However exchanging this data is insufficient, particularly in the management of critical facilities and it is recommended that they should be accompanied by exchanges concerning the related requirements (e.g. requirements about the equipment). Semantic Web technologies allow this association and make it possible to implement it more efficiently than the simultaneous implementation of different protocols (AP221, AP227 and AP233) of ISO 10303..

3. Constraints (such as those formulated in STEP Express language) can then be added to the OWL model. These constraints, expressed as additional rules, can be enriched by each stakeholder without having to be uniquely defined in the standard.

4. The general use of URIs allows the construction of dictionaries at normative for the all concepts, sector or private level.

5. The exchange of information can be done in OWL files which include both data and the data model. We can then speak of knowledge transfer.

6. The notion of façade ( $\mathrm{RDF}=$ Resource Description Framework) makes it possible to make data searchable via the Internet accessible using a suitable query system (SPARQL language). This language enables the concepts of the OWL data model in order to access the required information.

Another point to consider is that the exchanges are supported by the processes of each entity. In contractual terms, the exchanges must be traced and accompanied by a document possibly associated with an electronic signature of compliance. 
The ISO15926 standard supports all its promises, the question being to find the most effective tools and see how they can be deployed in practice. This has been explained and experimented as set out in Part 3 of this document.

In conclusion, the new information technologies, especially the Semantic Web are able to provide innovation in achieving exchange standards: they allow exchanges that are internal and external to the Company and are more flexible to implement. They also allow different contexts to be aggregated. The emerging ISO15926 standards based on these technologies is potentially able to take up a key position (especially in process industries) and must therefore be investigated and equipped while maintaining a broad view of possible extensions and technical and contractual implementation constraints.

\section{Experience Feedback from Industrial Implementation}

The AIRE platform (AREVA Interoperabilty Rich Environment or AREVA IRING Environment following the redesignation of ISO15926 by the IRING brand) was developed to experiment and industrialize the above principles.

Technologically, this platform has been designed to:

- Be compliant with the Semantic Web technology,

- Implement all parts of ISO15926 ${ }^{1}$,

- Take into account the constraints related to data privacy and cyber security.

And in functional terms, it has been designed to:

- Be open to taking into consideration the exchange of data from multiple domains either by extension of the Part 2 model of ISO15926 and the RDL dictionary, Part 4 , or by integrating other standards (e.g. - ISA, BIM/IFC, STEP).

- Support the information publishing process, filtering information to be published ${ }^{2}$, creation and management of publication spaces according to data and recipients ${ }^{3}$.

- Produce reports accompanying the publication of such information in order to meet contractual requirements.

- Support the information integration process with evaluation of changes between two successive integrations and performance of integrity checks ${ }^{4}$.

- Produce examination and integration reports to meet contractual requirements.

- Allow queries on data published by means of query generators.

- Exchange data sets by producing OWL files (on XML layer) making data and data model consistent with each other.

\footnotetext{
${ }^{1}$ It's mandatory to share data thru internet way, to extend the ISO15926 data model with new concepts and dictionary.

${ }^{2}$ To avoid publication of internal data that the company have to protect if these data are also stored in the application with the data that the company must publish.

${ }^{3}$ To separate information regarding recipient need to have the data.

${ }^{4}$ To control data with company rules before integration in the target tool.
} 
- Produce private RDLs and check correspondence (mapping) between source data and the RDLs (those maintained by certification bodies PCA/JORD and private RDLs generated) within a collaborative dialogue between two third-party partners. This check focuses on the completeness of the mapping and on the practical checks on the basis of test data before running the exchanges.

- Manage master data with alerting in case of modification of these data

- Take into account the exchange of attribute data, schematic data and ultimately 3D data and their changes (version or time effectiveness).

To demonstrate its capability, several connectors are made with off-the-shelf packages so as to build some real feedback on tool integration.

This has made it possible to validate the source integration methodology and to develop transactions and man-machine interfaces related to the above process: exchanges are prepared in AIRE but are directly carried out in the interfaces of these tools which therefore become interoperable.

\subsection{Source Integration Methodology}

The founding principle of the AIRE methodology (using the work described in [4]) is a) to transpose the data model of the application into an interface (source or target) and perform data transfer (import or export) in the formalism of the Semantic Web: b) construction of ontology of the application and c) execution of technical interface functions. This action gives a mirror of the data application in RDF format (in the form of subject-predicate-object triples) of both the data model and data directly manipulated by an OWL reasoner tool (into which the ontology of the standard has previously been loaded).

This is done once and for all for each application. The import and export functions can then be realized by the same way.

\subsection{Exchange Transformation Methodology in the Standard}

The second step is then to link the dictionary elements to the elements of the data model of the application. This is called "mapping" and is handled within a module of AIRE, the "configuration editor" which can question the public RDLs maintained by the PCA) or create private RDLs (at the level of the company, a group of companies or a business sector) ${ }^{5}$.

This operation is performed once per application and per standard. However, it needs to have a validation process to ensure that the mapping made is understood by the other parties: and the "configuration editor" can do this in collaborative mode with a third party, independently of whether the latter has the AIRE platform or not.

\footnotetext{
${ }^{5} \mathrm{RDL}=$ Reference Data Library $=$ the dictionary of the standard.
} 


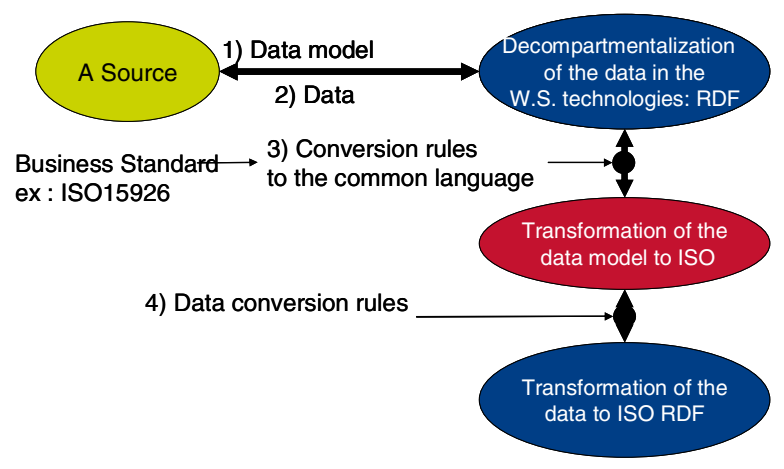

Fig. 4. Transformation principle

The configuration editor then generates a certain number of rules that are added to the ontology of the standard (here Part 2 of the ISO15926 standard) making it possible to link the dictionary (Part 4) and the roles (Part 7).

These rules are then carried out to publish or integrate the application data in interaction with the formalisms of the standard. This formalism can be expressed in the language of the Semantic Web (through RDF facades or OWL exports as is the case for ISO 15926), but can be expressed in other formats (XML based for example such as PROTEUS/XMPLANT or as AEX).

\subsection{Validation and Certification}

The AIRE solution has been validated with blind exchanges with a third party as part of a handover process. This experiment has improved functionality and means that the solution can be presented for certification by the PCA (POSC CAESAR ASSOCIATION).

\subsection{Feedback}

Firstly, the inclusion of a new standard in the company takes time that should not be underestimated. It seems important that the company should build case studies, depending on its activity and its own feedback, and clearly identify its levers, whether these be technical, technological, business or commercial.

In the case of the developments and experiments made, the flexibility provided by the Semantic Web is confirmed without significant performance degradation due to this flexibility.

One difficulty that should not be underestimated is related to communication of the standard and its implementation especially in the presentation of findings from the experiments: if it is too technical, it will seem very complicated and will alarm decision-makers; if this complexity is hidden, it becomes too easy or simplistic and states the obvious. It is therefore necessary to refer to difficulties encountered daily by the company which requires prior acceptance of these problems. 
Finally, the implementation of these interoperability tools introduces new tasks and new roles or may even require adaptation of the organization within the Company, particularly in the management of these interfaces with third parties.

\section{Conclusion and Extensions}

Developments and experiments carried out as part of the R\&D project led to the construction of an accomplished solution, benefiting both from the advantages of a solid base, and also from business transactions deriving from a group with both engineering and operation/maintenance of complex systems services.

It has also been shown that interoperability should be seen as a specific function with specialized stakeholders defining models and mappings, and organizing exchange flows.

This solution is integrated into the PLM repositories, which thereby have an additional interoperability function, and prevents/reduces manual copying of information or the implementation of specific point-to-point developments as well as the risk of errors and related cost overruns.

The choice of technological solutions and their design is essential in order to accompany these changes without calling them into question. The Semantic Web technologies and their implementation flexibility allow us to meet these goals by drastically reducing the costs and risks: they live up to their promises.

\section{References}

[1] NEI Nuclear Asset Management Community of Practice Report: The Standard Nuclear Performance Model - A Process Management Approach - Revision 4 et INPO AP929 Configuration Management Process Description

[2] Etude NIST (NIST GCR 04-867: Cost Analysis of Inadequate Interoperability in the U.S. Capital Facilities Industry) published in 2004 for an assessment of such costs (2004)

[3] Document " introduction to ISO15926" published by Fiatech in October 2011 for a description of the standard, its history and a detailed argument for this change

[4] Berners-Lee, T., Hendler, J., Lassila, O.: The Semantic Web. Scientific American 284(5), 34-43 (2001)

[5] Bellatreche, L., Pierra, G., Xuan, D.N., Hondjack, D., Ameur, Y.A.: An a Priori Approach for Automatic Integration of Heterogeneous and Autonomous Databases. In: Galindo, F., Takizawa, M., Traunmüller, R. (eds.) DEXA 2004. LNCS, vol. 3180, pp. 475-485. Springer, Heidelberg (2004)

[6] Topbraid, Editor of a toolbox for the Semantic Web, http://www. topquadrant. com/resources/documentation.html\#tbc

[7] Teijgeler, H.: The Process Industries and the ISO 15926 Semantic Web, http: / / www. infowebml.ws/intro/paper.htm

[8] Le Duigou, J., Bernard, A., Perry, N., Delplace, J.C.: Generic PLM system for SMEs: Application to an equipment manufacturer. IJPLM (2012)

[9] Matsokis, A.: An Ontology-Based Approach for Closed-Loop Product Lifecycle Management, Thesis (2010), http: / / infoscience.epfl.ch/record/150273/ files/EPFL_TH4823.pdf 